observational studies in this area also showed lower mortality in PCl-treated patients.

The investigators found few data on the use of adjunctive therapies in this setting. One study showed that low-molecular-weight heparin carried a high risk of intracranial hemorrhage when used in combination with tenecteplase in elderly patients; however, this may have been related to inappropriately high heparin dosing. Scanty data on the use of direct thrombin inhibitors in elderly patients undergoing thrombolysis show no survival benefit. One study suggested that bivalirudin was superior to unfractionated heparin in the prevention of reinfarction in older patients treated with streptokinase, although the drug was also associated with a higher rate of intracranial hemorrhage. Finally, a benefit of glycoprotein Ilb/IIla receptor antagonists as adjuncts to thrombolysis has yet to be demonstrated in the elderly; subgroup analyses have shown that these agents do not reduce mortality and might increase the risk of bleeding. In contrast, one study showed that abciximab improved outcomes in patients aged over 65 years when used as an adjunct to stent implantation during primary $\mathrm{PCl}$.

As a result of this analysis, and while more robust data are awaited, Mehta et al. have recommended an approach for the treatment of STEMI in patients aged $\geq 65$ years. This focuses on the use of rapid primary $\mathrm{PCI}$ where available, and efficient systems for transferring patients to appropriate treatment centers.

Original article Mehta RH et al. (2005) Reperfusion strategies for acute myocardial infarction in the eldery. J Am Coll Cardiol 45: 471-478

\section{ACE inhibitor therapy and cognitive function in heart failure patients}

Patients with heart failure often suffer cognitive impairment, probably because of chronic cerebral hypoperfusion, and this is associated with hastened mortality and disability. Zuccalà and co-workers asked whether this problem might be alleviated by angiotensin-convertingenzyme inhibitors (ACE inhibitors), which have been found to increase cerebral blood flow in subjects with left-ventricular dysfunction.

They analyzed data from a large, collaborative study of adverse drug reactions in hospitalized patients in Italy. Of 12,081 patients included in the analysis, 1,220 had been diagnosed with heart failure and none had received ACE inhibitors before hospitalization. Three domains of cognitive performance, namely information, memory and concentration, were assessed on admission and discharge using the Hodkinson Abbreviated Mental Test (AMT).

ACE inhibitor therapy was initiated after hospital admission in 446 (37\%) of the heart failure patients. Increased AMT scores on discharge were significantly more frequent in this subset of patients than among those who did not receive these drugs (30\% vs $22 \%$ for the two groups, respectively; $P=0.001$ ). In a multivariate analysis, ACE inhibitor therapy was independently associated with improved cognitive performance in patients with heart failure, irrespective of blood pressure levels (odds ratio 1.57, 95\% Cl 1.18-2.08). Higher doses of the drugs and longer duration of therapy increased the odds of improved AMT scores still further. Importantly, treatment with ACE inhibitors did not appear to affect cognitive performance among patients without heart failure.

Concluding that ACE inhibitor therapy "might selectively improve cognitive performance in patients with heart failure", the investigators draw attention to the importance of systematic neuropsychological assessment in this setting.

Original article Zuccalà G et al. (2005) Use of angiotensinconverting enzyme inhibitors and variations in cognitive performance among patients with heart failure. Eur Heart $J$ 26: $226-233$

\section{Repair of descending thoracic aortic aneurysm}

A recent study by Makaroun and colleagues has investigated the GORE TAG endoprosthesis (WL Gore \& Associates Inc., Flagstaff, AZ) for the treatment of descending thoracic aortic aneurysm (DTAA). The results suggest that the device is safe and is associated with a low rate of aneurysm-related death.

The first such device to reach phase II trials in the US, the GORE TAG endoprosthesis is a flexible, nitinol-supported expanded polytetrafluoroethylene tube of $26-40 \mathrm{~mm}$ diameter, available in three lengths. Under fluoroscopy and using standard endovascular techniques, 